\title{
Comparative metabolomic analysis of Sinorhizobium sp. C4 during the degradation of phenanthrene
}

\author{
Young Soo Keum • Jong Su Seo • Qing X. Li • \\ Jeong Han Kim
}

Received: 14 April 2008 / Revised: 16 June 2008 / Accepted: 18 June 2008 / Published online: 31 July 2008

(C) The Author(s) 2008

\begin{abstract}
Comparative metabolic responses of Sinorhizobium sp. C4 were investigated. Comprehensive metabolites profiles, including polar metabolites, fatty acids, and polyhydroxyalkanoates were evaluated through untargeted metabolome analyses. Intracellular metabolomes during the degradation of phenanthrene were compared with those from natural carbon sources. Principal component analysis showed a clear separation of metabolomes of phenanthrene degradation from other carbon sources. Shift to more hydrophobic fatty acid was observed from the analysis of fatty acid methyl ester. Polyhydroxyalkanoate from strain $\mathrm{C} 4$ was composed mainly with 3-hydroxybutyric acid and small amount of 3hydroxypentanoic acid, while the monomeric composition was independent on carbon sources. However, the amount of polyhydroxyalkanoates during degradation of phenanthrene was $50-210 \%$ less than those from other carbon sources. Among 207 gas chromatography-mass spectrometry peaks from the polar metabolite fraction, $60 \%$ of the peaks were identified and compared. Several intermediates in tricarboxylic acid cycles and glycolysis were increased during
\end{abstract}

Y. S. Keum·J. H. Kim $(\bowtie)$

Department of Agricultural Biotechnology,

Seoul National University,

San56-1, Silim-dong, Gwanak-Gu,

Seoul, Republic of Korea

e-mail: kjh2404@snu.ac.kr

\section{J. S. Seo}

Korea Institute of Toxicology,

19 Shinseong-ro, Yuseong-gu,

Daejeon, Republic of Korea

\section{Q. X. Li}

Department of Biosciences and Biogengineering,

University of Hawaii,

1955 East-West Road,

Honolulu, HI 96822, USA phenanthrene degradation. Accumulation of trehalose was also evident in the phenanthrene-treated bacterium. Some amino acid, including branched amino acids, glycine, homoserine, and valine, were also increased, while more than $70 \%$ of identified metabolites were decreased during the phenanthrene metabolism. Accumulation of sulfur amino acids and nicotinic acid suggested the possible oxidative stress conditions during phenanthrene metabolism.

Keywords Sinorhizobium · Polycyclic aromatic hydrocarbon · Metabolomics · Polyhydroxyalkanoate . Fatty acids

\section{Introduction}

Rapid accumulation of genomic information provides detailed understanding of specific organisms and/or biochemical pathways. Currently, genomes of more than 200 organisms have been fully uncovered. Genomic information of several bacterial species with xenobiotics degradation activities are also available, including Arthrobacter aurescens TC1, Burkholderia xenovorans LB400, several species in the genus of Mycobacterium, Novosphingobium aromaticivorans DSM1244, and Sphingomonas wittichii RW1. System-wide analyses of these bacteria have been performed in the context of proteomics or genomics (e.g., Denef et al. 2004, 2005; Ishii et al. 2007; Kweon et al. 2007). In consideration of the fact that the biological systems are extremely complex array of interaction between genes, proteins, and metabolites, detailed information of metabolomic differentiation is also one of the most important prerequisite for better understanding of bacterial metabolism of environmental contaminants. Polycyclic aromatic hydrocarbons (PAHs) and polychlorinated biphenyls 
(PCBs) are ubiquitous contaminants which originated from various industrial processes. Because of their diverse toxicity profiles, PAHs and its metabolites pose a serious risk to the natural environment and humans (Armstrong et al. 2004; Eom et al. 2007). Among the rhizobial microorganisms, several bacterial species in the genus of Agrobacterium, Bradyrhizobium, Rhizobium, and Sinorhizobium are able to utilize PAHs, PCBs, or aromatic heterocycles or at least are tolerant to these toxic chemicals (Aitken et al. 1998; Damaj and Ahmad 1996; Frassinetti et al. 1998). Recent biochemical studies and metabolic profiling also revealed that some rhizobiales bacteria have corresponding genes and the potential to metabolize PAHs (Frassinetti et al. 1998; Galibert et al. 2001; Keum et al. 2006). Although there were high degrees of amino acid or nucleotide sequence diversities in degradative enzymes, only a limited number of PAH metabolites can be incorporated into primary metabolism (e.g., carbon dioxide, pyruvate, salicylate, gentisate, and protocatechuate from naphthalene, phenanthrene, and pyrene). In addition, several metabolites are toxic to microorganisms through the depletion of reducing equivalents or cofactors (Chang and Zylstra 1999; Kim et al. 2004). Rapid metabolic adaptation to environmental challenges is common in microorganism (e.g., Aeckersberg et al. 1998; Parnell et al. 2006). It can be expected that similar changes may also be observed in PAH-degrading bacteria. Quantitative and comparative information of the metabolomic differentiation will provide better understanding of bacterial PAH metabolism and give insight to improve the metabolic network for bioremediation of environmental contamination.

Sinorhizobium sp. C4, which can utilize phenanthrene as a sole carbon source for growth, was isolated from PAHcontaminated soils in Hawaii, USA (Keum et al. 2006). Detailed metabolite profiling proved that strain $\mathrm{C} 4$ can degrade phenanthrene through dioxygenase-catalyzed ring opening and formation of $o$-hydroxynaphthoate and phthalate (PHT; Keum et al. 2006). In this study, metabolite profiles of strain C4 during the degradation of phenanthrene were compared with those from natural carbon sources, common in primary metabolism.

\section{Materials and methods}

Culture of bacterium

Strain C4 was cultivated in nutrient broth (NB) or minimal salt medium (MM, Bastiaens et al. 2000), supplemented with several different carbon sources (phenanthrene, glucose, or sodium pyruvate, $300 \mathrm{mg} / \mathrm{L}$, respectively) at $30^{\circ} \mathrm{C}$. Cells were harvested at mid-log phase of exponential growth where the optical densities at $600 \mathrm{~nm}$ reached approximately 1.0 .

\section{Chemical}

The following reagents were obtained from Aldrich (Sigma-Aldrich Korea, South Korea): phenanthrene, sodium pyruvate, methoxylamine hydrochloride, pyridine, bis-(trimethylsilyl)trifluoroacetamide-trimethychlorosilane (BSTFA-TMCS), and poly(3-hydroxybutyric acid [3HB]co-3-hydroxyvaleric acid). Standards of primary metabolism were obtained from Aldrich or TCI. Methanol and other solvents were high-performance liquid chromatography grade or higher.

Analysis of metabolites of phenanthrene

After 3-7 days, cultures were centrifuged at $4,000 \times g$ for $15 \mathrm{~min}$. Phenanthrene metabolites in culture supernatant $(500 \mathrm{ml})$ were extracted according to the literature method (Keum et al. 2006). After removal of organic solvent, BSTFA-TMCS $(150 \mu \mathrm{l})$ was added to a suspension of the residue in dry pyridine $(400 \mu \mathrm{l})$ and derivatized at $80^{\circ} \mathrm{C}$ for $2 \mathrm{~h}$. Aliquot amount of samples were analyzed with gas chromatography-mass spectrometry (GC-MS). Detailed conditions for GC-MS were similar with that of polar metabolites and described in another section. Intracellular phenanthrene metabolites were determined as follows: cell pellet $(500 \mathrm{mg})$, suspended in acetone $(10 \mathrm{ml})$, was disrupted by sonication (550 Sonic Dismembrator, Fisher Scientific; $1 \mathrm{~min}$ pulse and $2 \mathrm{~min}$ cooling, four cycles). After centrifuge, cellular debris was further extracted with methanol $(10 \mathrm{ml})$ and ethyl acetate $(10 \mathrm{ml})$. Combined organic extracts were dried under gentle stream of air. The residues were analyzed through the same procedures of extracellular metabolites.

Extraction and derivatization of polar metabolites

Cell pellet was washed with distilled water. Bacterial cells (200 $\mathrm{mg}$ ) were suspended in $70 \%$ methanol $(15 \mathrm{ml})$ and disrupted by sonication (550 Sonic Dismembrator, Fisher Scientific; 1 min pulse and 2 min cooling, eight cycles). Cellular debris was removed by centrifugation $(15,000 \times g$, $10 \mathrm{~min}$ ), and the supernatant was dried under vacuum. The residue was resuspended in $500 \mu$ of dry pyridine, derivatized with $100 \mu \mathrm{l}$ of methoxylamine $(100 \mathrm{mg}$ methoxylamine hydrochloride in $10 \mathrm{ml}$ pyridine) for $1 \mathrm{~h}$, and subsequently with $200 \mu \mathrm{l}$ of BSTFA-TMCS for $2 \mathrm{~h}$ at $80^{\circ} \mathrm{C}$.

Fatty acid methyl ester analysis

Medium to long chain fatty acid profiles were determined by MIDI protocols (Sasser 2006). Briefly, cell pellet (100 mg) was saponified with methanolic potassium hydroxide, 
followed by trans-esterification and extraction of Fatty acid methyl esters (FAMEs) with a mixture of hexane and methyl $t$-butyl ether. Aliquot amount of extracts was analyzed with GC-MS. The operation condition was similar with polar metabolites.

\section{Polyhydroxyalkanoate analysis}

To determine the monomeric composition of polyhydroxyalkanoates (PHAs) from strain C4, PHAs were purified by the previous method (Berger et al. 1989). In brief, the cell pellet (2 g) was treated with a solution of sodium hypochlorite $\left(20 \mathrm{ml}, 30 \mathrm{~min}, 50^{\circ} \mathrm{C}\right)$. Resulting suspensions were centrifuged $(15,000 \times \mathrm{g}, 20 \mathrm{~min})$. Pellet was subsequently washed with distilled water, methanol, and acetone. Dry pellet was extracted with boiling chloroform $(5 \mathrm{ml})$, and PHAs were precipitated with cold ethanol $(20 \mathrm{ml})$. Aliquot amount of precipitates were hydrolyzed with methanolic potassium hydroxide and extracted with ethyl acetate after acidification. Monomeric hydroxyalkanoates were derivatized with BSTFA-TMCS and analyzed with similar GC-MS procedures with that of polar metabolites.

PHA contents in strain C3, grown in different carbon sources, were determined in small scale (100-mg cell pellets). Bacterial cells were saponified by the similar method of FAME preparation. The suspension $(5 \mathrm{ml})$ was acidified with small amount of concentrated hydrochloric acid and diluted with saturated aqueous sodium chloride solution $(10 \mathrm{ml})$. PHA monomers were extracted with methyl $t$-butyl ether $(20 \mathrm{ml} \times 2)$. After removal of the solvent, the residue was mixed with dry pyridine $(400 \mu \mathrm{l})$ and BSTFA-TMCS $(100 \mu \mathrm{l})$ and followed by derivatisation at $80^{\circ} \mathrm{C}$ for $1 \mathrm{~h}$.

\section{Instrumental analysis}

Metabolite profiles were analyzed with GC-MS (Shimadzu GCMS QP-2000 and GC-2010), equipped with DB-1 column $(60 \mathrm{~m}, 0.25 \mu \mathrm{m}$ film thickness, $0.2 \mathrm{~mm}$ inner diameter; Agilent Technologies, USA). Helium was a carrier gas at a flow rate of $1 \mathrm{ml} / \mathrm{min}$. The column temperature for polar metabolites and PHA analyses were as follows: $95^{\circ} \mathrm{C}(10 \mathrm{~min})$ and raised to $295^{\circ} \mathrm{C}$ at a rate of $2^{\circ} \mathrm{C} / \mathrm{min}$ and held for $20 \mathrm{~min}$. For FAME analysis, the column temperature was as follows: $50^{\circ} \mathrm{C}(10 \mathrm{~min})$, raised to $280^{\circ} \mathrm{C}\left(2.5^{\circ} \mathrm{C} / \mathrm{min}\right)$, and held for $20 \mathrm{~min}$. Injector and interface temperatures were held at $270^{\circ} \mathrm{C}$ and $280^{\circ} \mathrm{C}$, respectively. The mass spectrometer was operated at electron impact mode at $70 \mathrm{eV}$.

\section{Data analysis}

Chromatographic data generated from GC-MS were converted to netCDF file and imported into the metAlign software (http://www.metAlign.nl) to align and correct the background of the chromatograms. Chemical identities of each peak and area were determined by GCMS Solution software with implemented Wiley mass spectral library (Dongil-Shimadzu, South Korea). Before the statistical analyses, peak area of each metabolite was normalized with average value of overall sample set. Descriptive statistics, principal component analysis (PCA), and other statistical treatment were performed with SAS package (SAS Korea, South Korea) or SIMCA-P (Umetrics, Sweden).

\section{Results}

Growth of bacterium and phenanthrene metabolism

Various carbon sources, including NB, glucose, pyruvate, and phenanthrene, can support the bacterial growth. In natural carbon source media (NB, glucose, and pyruvate), $\mathrm{C} 4$ grew without the lag phase and reached to the stationary phase after 7-8 days, while the cells grown with phenanthrene as the sole carbon source showed a short lag period, followed by exponential growth and reached to the stationary phase after 10 days (Fig. 1). Bacterial cells for the metabolomic study were collected after 3 (NB, glucose, and pyruvate) or 7 days (phenanthrene), and the phenanthrene metabolites in the culture medium were determined at the same time.

Several metabolites of phenanthrene were detected in the culture medium (Fig. 2). Among the metabolites, PHT (retention time, Rt, $30.72 \mathrm{~min}$ ), 1-hydroxy-2-naphthoate (Rt, $39.41 \mathrm{~min}$ ), and 4-(1-hydroxy-naphthalen-2-yl)-2-oxobut-3-enoate (HNB, Rt, $52.07 \mathrm{~min}$ ) were the most abundant metabolites at the sampling time for intracellular metabolomics (7 days). Other metabolites, including protocatechuate (Rt $43.57 \mathrm{~min}$ ), gentisate (Rt $45.73 \mathrm{~min}$ ), and phenanthrene diols (Rts, 61.08 and $61.34 \mathrm{~min}$ ), were also detected in minor quantities. These metabolite profiles coincided with the previous results (Keum et al. 2006). Among the above extracellular phenanthrene metabolites, only trace amounts of PHT and phenanthrene were found in cellular extracts (data not shown).

Polar metabolite profile

Overall, more than 300 peaks were observed in samples (Fig. 3). After aligning peaks to reference GC-MS chromatograms (glucose-grown cells), each peak was evaluated to identify impurities from experimental procedures. The peak(s) highly deviated from other samples in the same batch were removed from the statistical analysis. In addition, peaks from incomplete derivatization (e.g., 2- 


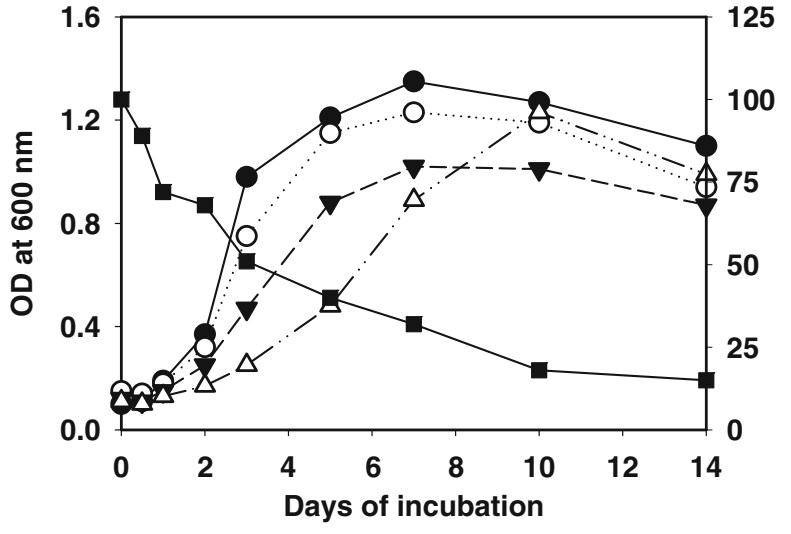

Fig. 1 Growth of Sinorhizobium sp. C4 in nutrient broth, phenanthrene, glucose, and pyruvate in minimal salt medium and the degradation of phenanthrene. Filled squares, percent of phenanthrene remaining; optical density at $600 \mathrm{~nm}$, filled circles, nutrient broth; open circles, glucose in MM; filled triangles, pyruvate in MM; open triangles, phenanthrene in $\mathrm{MM}$

trimethylsyl [TMS] derivative of lysine vs. 3-TMS derivative) were also identified through the comparison of GCMS of corresponding standard metabolites. Approximately 207 metabolites were identified from the above analysis, and 118 metabolites were confirmed with standard metabolites or tentatively identified by mass spectral library search (Table 1). Identified metabolites were comprised with 30 organic acids, 34 amino acids, acyl amino acids, and aminoalkanoic acids, five nucleotides, 12 sugars and phosphates, 19 alcohols, including sugar alcohols, 11 alkylamines and phosphatidylamines, and seven cofactors and miscellaneous compounds. In general, larger numbers of different metabolites were observed in NB- and glucosegrown cells (115 and 108 metabolites, respectively) than those from pyruvate- or phenanthrene-supplemented cultures (93 and 78 metabolites, respectively). The contents of sugars or sugar phosphates, especially hexose phosphates (e.g., glucose-6-phosphate), were higher in the glucosesupplemented culture, while free pentose concentrations were relatively higher in the cultures of different carbon sources (NB, pyruvate, and phenanthrene). For example,

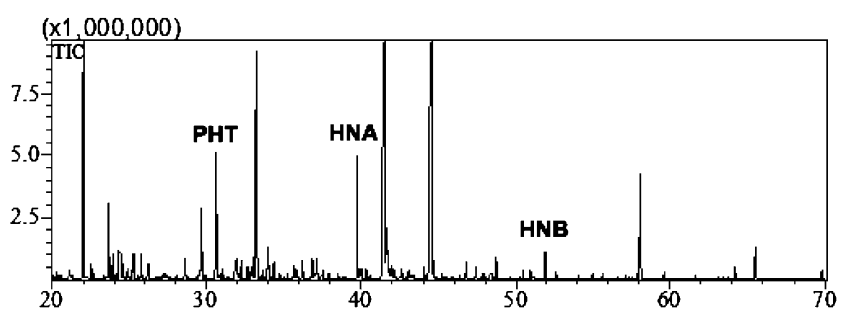

Fig. 2 GC-MS chromatograms of phenanthrene metabolites by Sinorhizobium sp. C4, grown in mineral salt medium with phenanthrene. Abbreviations: PHT phthalate, HNA 1-hydroxy-2-naphtoate, $H N B$ 4-(1-Hydroxy-naphthalen-2-yl)-2-oxo-but-3-enoate. Bacterial cells were harvested from the culture after 7 days

\section{a}

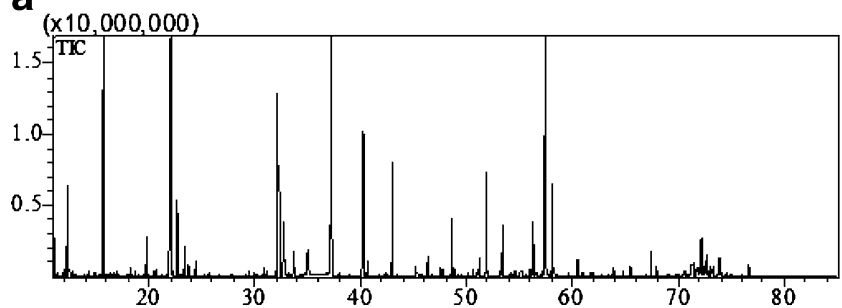

b

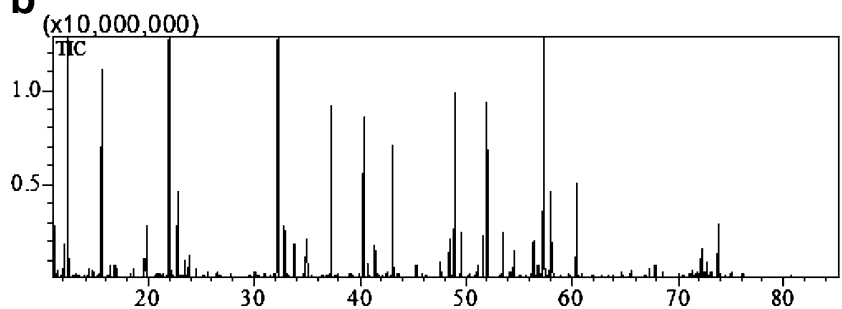

Fig. 3 Representative GC-MS chromatograms of intracellular polar metabolites of Sinorhizobium sp. C4, grown in nutrient broth (a) and minimal salt medium, supplemented with phenanthrene (b). Bacterial cells were harvested from the culture after 3 (glucose) or 7 days (phenanthrene)

ribose and ribose-5-phosphate concentrations were much higher in phenanthrene-supplemented culture than those of other carbon sources (Fig. 4a). High concentration of trehalose (Rt, 74.1 min, Fig. 3), which is the most abundant intracellular metabolites in Mycobacterium spp., was also detected in high quantities ( $1-2 \%$ of total metabolites). Other disaccharides, including lactose and maltose, were also detected in polar metabolite fractions. However, because of their high variation throughout the experimental batches, maltose was excluded from the statistical analyses. Several amino acids were also differentially accumulated or decreased, depending on the carbon sources (Fig. 4b,c). For example, higher concentrations of lysine, methionine, and glutamic acid were observed in glucose-, pyruvate-, or phenanthrene-supplemented cultures than those from complex media, while the level of serine is relatively lower in the single-carbon source-based culture, except phenanthrene. It is noteworthy that cysteine, another sulfur amino acid, was decreased in the phenanthrene-supplemented culture $(50 \%$ reduction in comparison with other carbon sources). In addition, the concentration of branched amino acid (e.g., valine, leucine, and isoleucine) was also dependent on the carbon sources. Among the cultures in $\mathrm{MM}$, higher extent of valine accumulation was observed than other carbon sources, while the level of leucine did not change (Fig. 4c). A significant increase in nicotinic acid was observed in phenanthrene-supplemented culture, while the level of $\beta$-alanine was reduced (Fig. 4d). The differential accumulation pattern of organic acids, related to numerous primary metabolic pathways, was found, including metabolites in glycolysis, tricarboxylic acid (TCA) cycle, pyruvate metabolism, propanoate, butanoate, 
Table 1 List of confirmed or tentatively identified polar metabolites in Sinorhizobium sp. C4, grown in different carbon sources ${ }^{\mathrm{a}}$

\section{Metabolites}

Organic acids

Amino acids
Malonic acid

Pyruvic acid

Lactic acid

Hexanoic acid

Glycolic acid

2-Hydroxybutyric acid

Oxaloacetic acid

Hydracrylic acid

4-Methoxybutyric acid

3-Hydroxyisovaleric acid

Benzoic acid

2-Hydroxyisocaproic acid

Succinic acid

Glyceric acid

Nonanoic acid

Glutaric acid

Malic acid

2-Hydroxyglutaric acid

Hydroxyalkanoic acid

4-Hydroxybenzoic acid

Tartaric acid

5-Ketogluconic acid

$\alpha$-Ketogluconolactone

$\beta$-Glycerophosphate

$\alpha$-Phosphoglycerate

2-Phosphoglycerate

Shikimic acid

$\alpha$-Ketoglutaric acid

$\alpha$-Keto-hydroxyalkanoic acid

Glyceric acid deriv.

Alanine

$\beta$-Alanine

Aspartic acid

Cysteine

Cystine

Glutamic acid

Glycine

Homoserine

Homocysteine

Hydroxyproline

Hydroxyproline deriv.

Isoleucine

Leucine

Lysine

Methionine

Phenylalanine

Proline

Proline deriv.

Pyroglutamic acid

Serine

Threonine

Tyrosine

Valine

$N$-Acetylamino acid

$\mathrm{N}$-Acetylaspartate

$\mathrm{N}$-Acetylglutamic acid

$\mathrm{N}$-Formylglycine

$\mathrm{N}$-Acetylglycine
Table 1 (continued)

Metabolites

\begin{tabular}{|c|c|}
\hline & $N$-Acylglycine \\
\hline & Glycine deriv. \\
\hline & Lysine deriv. \\
\hline & 2-Aminobutyric acid \\
\hline & $b$-Aminoisobutyric acid \\
\hline & Aminomalonic acid \\
\hline \multirow[t]{5}{*}{ Nucleotides } & Uracil \\
\hline & Thymine \\
\hline & Thymine deriv. \\
\hline & Uridine \\
\hline & Uridine deriv. \\
\hline \multirow[t]{12}{*}{ Sugar/phosphate } & Erythrose \\
\hline & Pentose \\
\hline & Ribose \\
\hline & Ribose-5-phosphate \\
\hline & Deoxyhexose \\
\hline & Fructose \\
\hline & Mannose \\
\hline & Galactose \\
\hline & Glucose \\
\hline & Glucose-6-phosphate \\
\hline & Trehalose \\
\hline & Lactose \\
\hline \multirow[t]{12}{*}{ Alcohol/sugar alcohol } & Erythritol \\
\hline & Xylitol \\
\hline & Inositol \\
\hline & Sugar alcohol $(7)^{\mathrm{b}}$ \\
\hline & 2-Methyl-1,2-propanediol \\
\hline & Phenethyl alcohol \\
\hline & 2,5-Hexadien-1,3-diol \\
\hline & Alkanol (2) \\
\hline & Diethylene glycol \\
\hline & Glycerol \\
\hline & Phosphoglycerylglycerol \\
\hline & Octadecenol \\
\hline \multirow[t]{11}{*}{ Amines } & Ethylamine \\
\hline & Allylamine \\
\hline & Ethanolamine \\
\hline & Aminoalkanol \\
\hline & Phosphatidylethanolamine \\
\hline & Phosphorylethanolamine-isothiocyanate \\
\hline & Phenylethanolamine deriv. \\
\hline & Triethanolamine \\
\hline & Phenylethanolamine \\
\hline & Phosphoethanolamine \\
\hline & Phosphatidylethanolamine deriv. \\
\hline \multirow[t]{7}{*}{ Cofactors and others } & Nicotinic acid \\
\hline & Nicotineamide \\
\hline & Pipecolic acid \\
\hline & 6-Acetyl-2,5-dihydroxy-1,4-naphthoquinone \\
\hline & Cadaverine \\
\hline & Citruline \\
\hline & Polyamine \\
\hline
\end{tabular}

${ }^{\text {a Among }} 207$ possible metabolites, only confirmed or tentatively identified metabolites which increase or decrease more than $50 \%$ between carbon sources and statistically significant are listed.

${ }^{\mathrm{b}}$ Numbers of peaks of different retention time, where the mass spectra showed typical fragmentation pattern of the corresponding class of metabolites 
Fig. 4 Differential accumulation of selected sugars and sugar phosphates (a), branched amino acids (b), and other selected amino acids (c) and cofactors and its precursors (d). Abbreviations: $N B$ nutrient broth, $G l u$ glucose, $P V$ pyruvate, PHE phenanthrene in MM, sulfur $A A$ sulfur-containing amino acids, including methionine, cysteine, and others. Error bar stands for standard deviation. Bacterial cells were harvested from the culture after 3 (glucose) or 7 days (phenanthrene)

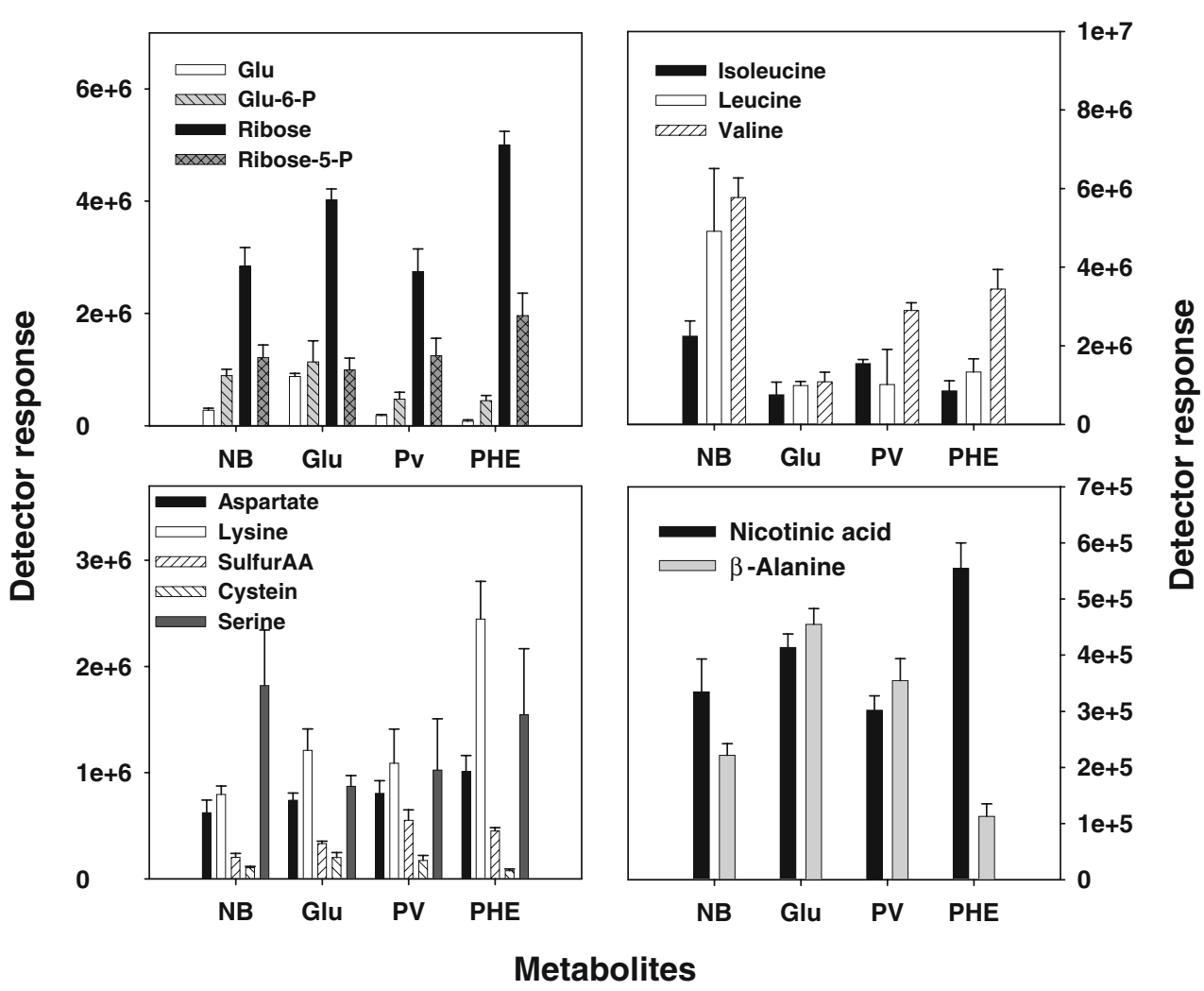

and C5 acid metabolism, glyoxylate shunt, glycerophospholipid metabolism, and branched amino acid biosynthesis. In general, a higher accumulation of some intermediates in glycolysis (e.g., 2-phosphoglycerate) and TCA cycle (e.g., oxalate) was found in the phenanthrene-supplemented culture (Fig. 5). However, a reduced level of other metabolites in the same metabolic pathways was also observed (e.g., 3phosphoglycerate, malate, and $\alpha$-ketoglutarate, Fig. 5). Other intermediates, including 2-hydroxyglutarate, malonate, and 3-hydroxyisovalerate, were also increased in phenanthrenesupplemented cultures.

Fatty acids and polyhydroxyalkanoate profiles

The summed peak area of medium to long chain fatty acids (C12 to C20) was slightly changed between different carbon sources (e.g., Fig. 6). However, a large difference has been found in relative composition of individual fatty acids. For example, a relative proportion of peak area of the peak (Rt $65.5 \mathrm{~min}$ ) was changed from 4-7\% (pyruvate, glucose, and NB) to $12.3 \%$ (phenanthrene). This fatty acid was tentatively identified as cyclopropyl-fatty acid (C18). Several 2- or 3-hydroxylated fatty acids were found in saponifiable lipid, including 3-hydroxytetradecanote, 3hydroxyhexadecanoate (3OH-C16), 3-hydroxyoctadecanoate, and 2- or 3-hydroxyeicosanoate (OH-C20). In comparison with other fatty acids, slight changes have been detected for these fatty acids, where the level of $3 \mathrm{OH}-\mathrm{C} 16$ decreased with concomitant increase in $3 \mathrm{OH}-\mathrm{C} 20$ in the phenanthrenesupplemented culture.

GC-MS analysis of purified PHAs from strain C4 showed approximately three peaks with characteristic MS
Fig. 5 Differential accumulation of selected organic acids in glycolysis (a) and TCA cycle (b). Abbreviations: $N B$ nutrient broth, Glu glucose, $P V$ pyruvate, $P H E$ phenanthrene in MM, 2PG 2phosphoglycerate, 3PG 3phosphoglycerate, LacticA lactic acid, $a-K G \alpha$-ketoglutarate. Error bar stands for standard deviation. Bacterial cells were harvested from the culture after 3 (glucose) or 7 days (phenanthrene)
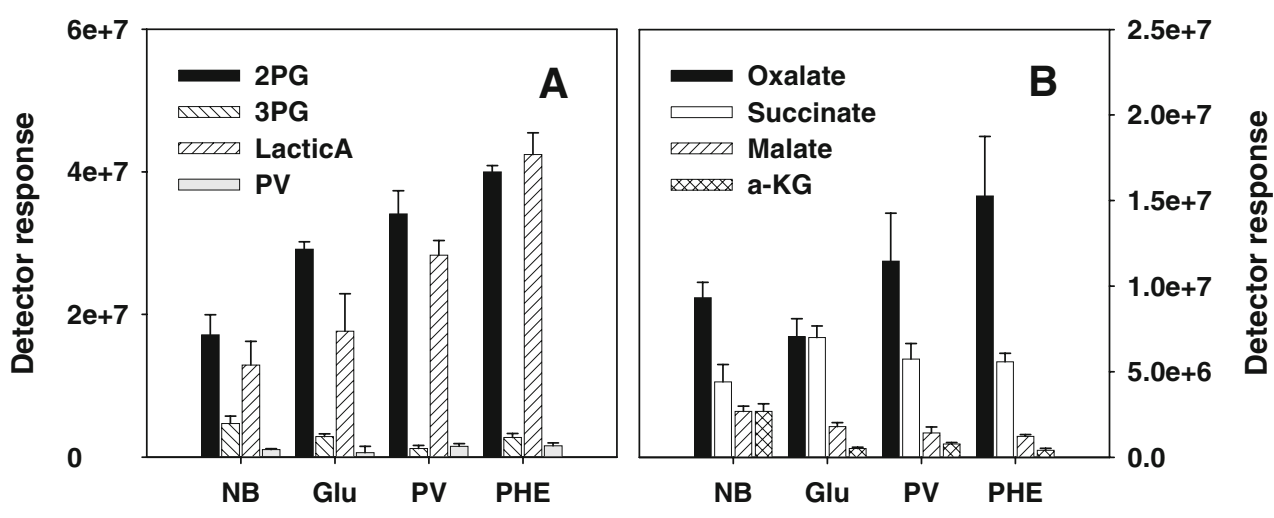
a

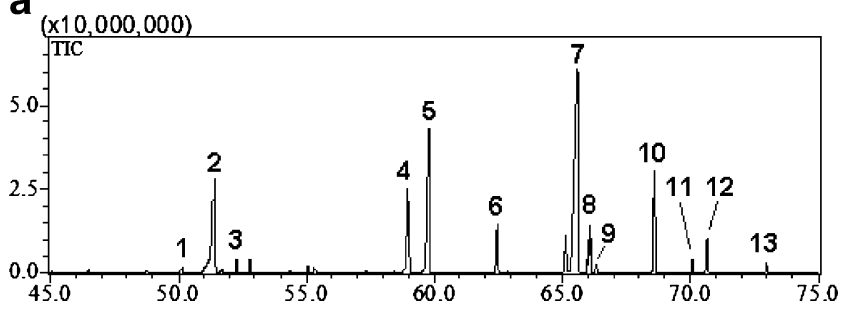

b

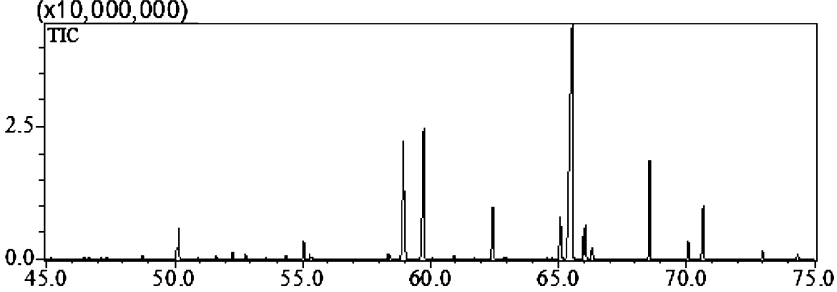

Fig. 6 Representative GC-MS chromatograms of medium to long chain fatty acids of Sinorhizobium sp. C4, grown in minimal salt medium, supplemented with glucose (a) and phenanthrene (b). Bacterial cells were harvested from the culture after 3 (glucose) or 7 days (phenanthrene). Numbers of fatty acids were for: 1, 3hydroxydodecanoic acid; 2 , tetradecanoic acid; 3 , unknown fatty acid; 4, 3-hydroxytetradecanoic acid; 5, hexadecanoic acid; 6, methylhexadecanoic acid; 7, cyclopropane-octanoic acid; 8 , octadecenoic acid; 9, octadecanoic acid; 10 , methyloctadecanoic acid; 11 , nonadecanoic acid; 12, 3-hydroxyoctadecanoic acid; 13, Eicosanoic acid

fragmentation $(\mathrm{m} / \mathrm{z}$ 147) of TMS derivatives of hydroxyalkanoic acids. The corresponding peaks were identified as lactic acid (HL, Rt $15.72 \mathrm{~min}$ ), 3HB (Rt $20.23 \mathrm{~min}$ ), and 3-hydroxypentanoic acid (3HP, Rt $24.65 \mathrm{~min}$ ), respectively (Fig. 7). In general, monomeric composition of PHAs was independent of the carbon sources (Table 2). Although medium- to long-chain 2- or 3-hydroxyfatty acid were detected in appreciable quantities during fatty acid analysis, similar peaks were not detected in purified PHA samples, indicating that $\mathrm{HL}, 3 \mathrm{HB}$, and $3 \mathrm{HP}$ were the only monomeric units in PHAs, where 3HP was the most abundant monomer from strain $\mathrm{C} 4$.

\section{Discussions}

As a new tool of system biology, comprehensive metabolite profiling or metabolomics has been studied in various scientific applications, including medicinal chemistry, plant physiology, and microbiology (e.g., Mashego et al. 2007; Robertson 2005; Weckwerth 2003). In comparison with the other applications, relatively limited numbers of publications are available for microbial metabolomics. Diversities in microorganisms and their growth environment may prevent more detailed metabolomic research for specific microorganisms. Many genetically modified microorganisms have been developed for various purposes. However, the application of such organisms on environmental issues (e.g., a

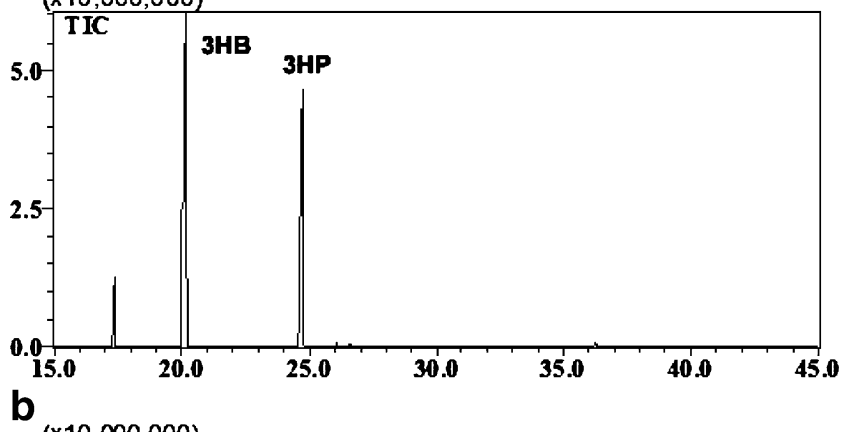

$(x 10,000,000)$

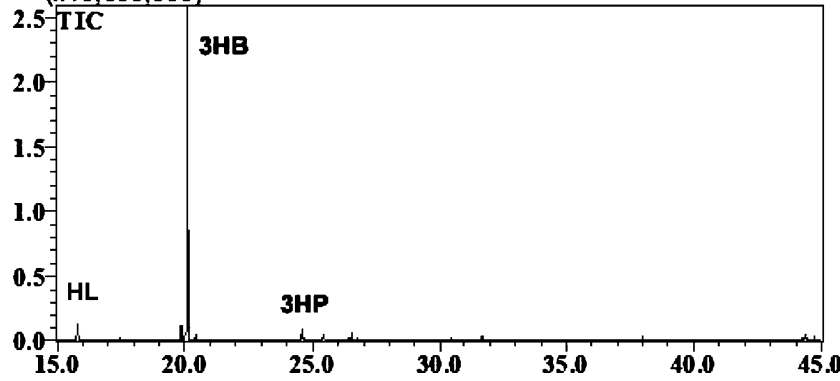

Fig. 7 GC-MS chromatograms of monomers prepared from standard polyhydroxyalkanoate from Aldrich, poly(3-hydroxybutyric acid-co3-hydroxypentanoic acid) (a) and from Sinorhizobium sp. C4, grown in mineral salt medium with phenanthrene (b). Abbreviations: $H B$ hydroxybutyric acid, HP 3-hydroxypentanoic acid

bioremediation) was usually unsuccessful since the transgenic mutants or wild-type microorganisms are not compatible to specific environments (Case and de Lorenzo 2005). More comprehensive understanding based on system biology will be much more helpful to solve these problems. Among the rhizobial bacteria, comprehensive profiles or reference maps of intracellular metabolites of Sinorhizobium meliloti became available recently (Barsch et al. 2004). Because of their possible symbiosis with plants, rhizobial bacteria with xenobiotic-degrading activity are important for the application of bioremediation and phytoremediation. As mentioned above, microorganisms under harmful environment may

Table 2 Hydroxyalkanoic acid concentration and composition of polyhydroxyalkanoates from Sinorhizobium sp. C4, grown with different carbon sources

\begin{tabular}{llc}
\hline $\begin{array}{l}\text { Carbon } \\
\text { sources }\end{array}$ & $\begin{array}{l}\text { Composition } \\
(3 \mathrm{HB} / 3 \mathrm{HP} / \mathrm{HL})^{\mathrm{a}}\end{array}$ & $\begin{array}{l}\text { Concentration } \\
(\mathrm{mg} / \mathrm{g} \text { wet weight })^{\mathrm{b}}\end{array}$ \\
\hline $\begin{array}{l}\text { Nutrient broth } \\
\text { Glucose }\end{array}$ & $93 / 2 / 10$ & $55.2 \pm 9.4$ \\
Pyruvate & $93 / 2 / 8$ & $85.3 \pm 8.9$ \\
Phenanthrene & $90 / 10 / 2$ & $45.5 \pm 5.1$ \\
\hline
\end{tabular}

${ }^{\text {a }}$ Ratio of 3HB 3-hydroxybutyric acid, 3HP 3-hydroxypentanoic acid, and $H L$ lactic acid

${ }^{\mathrm{b}}$ Concentration was calculated according to the bases of the concentration of 3-hydroxybutyric acid, released by alkali hydrolysis 
adapt themselves to corresponding stress through comprehensive reprogramming of a wide array of primary metabolism and possibly secondary metabolism, which results in largely different metabolite profiles. More detailed understanding of these responses will provide valuable information to improve specific bacterium for environmental application. In overall, PCA showed a clear separation of intracellular metabolite profiles from different carbon sources (Fig. 8). Because of the possible requirement of adaptation to different carbon sources, large changes were anticipated in intracellular metabolomes.

Bacterial metabolism of $\mathrm{PAH}$, where high concentration of PHT is produced, may pose a serious problem to PAHdegrading bacterium, itself. Because PHT, a structural homologue of quinolinate, can competitively inhibit quinolinate phosphoribosyltransferase, rapid accumulation of PHT may decrease intracellular pyridine nucleotide level, especially nicotinamide adenine dinucleotide (NAD). Chang and Zylstra reported two different quinolinate phosphoribosyltransferases from a strong PHT-degrading Burkholderia, where one of the enzymes was inducible by PHT, and its mutation resulted in impaired growth in a PHT-supplemented culture (Chang and Zylstra 1999). In addition to its role in numerous primary metabolisms, NAD also participates in PAH degradation as a reducing equivalent of PAH dioxygenase and monooxygenase. Elevated levels of nicotinic acid and nicotineamide in strain

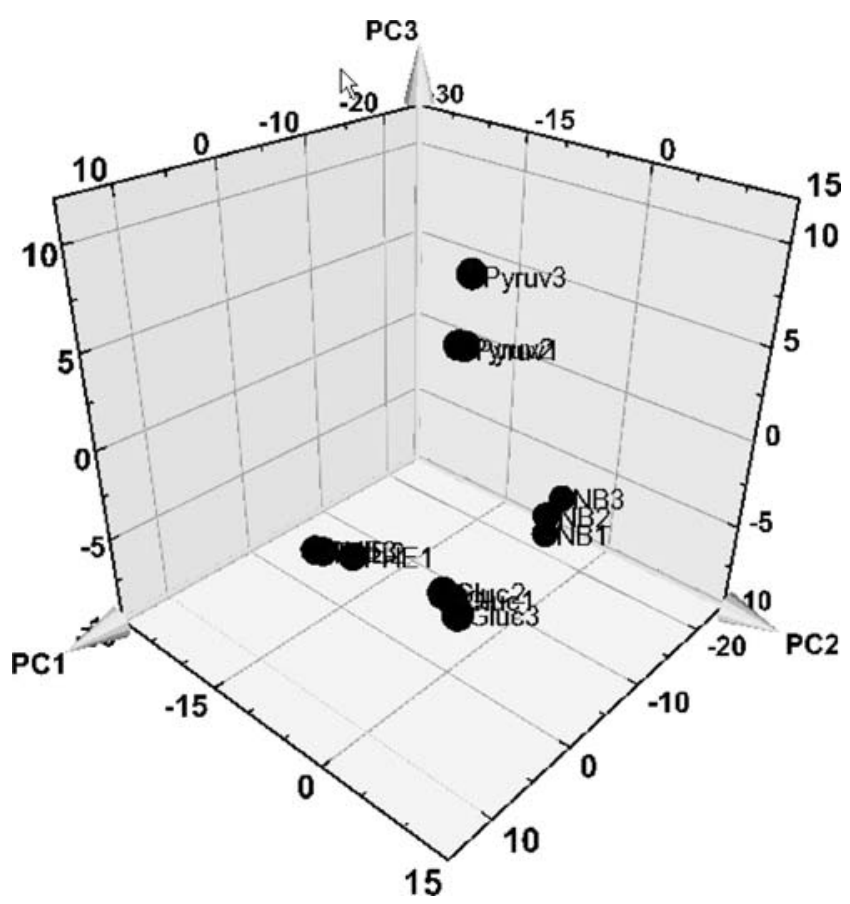

Fig. 8 PCA score plot of polar metabolites of Sinorhizobium sp. C4, grown in different carbon sources. Abbreviations: $N B$ nutrient broth, Gluc glucose in MM, Pyruv pyruvate in MM, PHE phenanthrene in MM. Cumulative eigenvalues of $\mathrm{PC} 1, \mathrm{PC} 2$, and $\mathrm{PC} 3$ are $0.65,0.81$, and 0.87
C4 during phenanthrene metabolism indicate the higher requirement of NAD biosynthesis for PAH degradation and primary metabolism. Accumulation of aspartate may also support such explanations since the amino acid is easily transformed into quinolinate. The reduction of $\beta$-alanine concentration was particularly important since it is a direct precursor of coenzyme A (CoA), which, in turn, is a precursor of numerous primary metabolism. It is noteworthy that aspartate decarboxylase, a key enzyme of $\beta$-alanine biosynthesis from aspartate, is slowly inhibited by a high concentration of aspartate (Scott et al. 2007). Another possible reasons of $\beta$-alanine depletion includes rapid consumption to other metabolites namely, CoA biosynthesis. Fatty acid biosynthesis requires large portion of $\mathrm{CoA}$ as acyl-CoA. However, it was not clear which metabolic factors have stronger effects over $\beta$-alanine pools.

In addition to PHTs, several downstream metabolites of PAHs may also be toxic to microorganisms. For example, catechol-type metabolites have an inhibitory effect against $\mathrm{PAH}$ dioxygenase, which is a first enzyme in PAH metabolism (Vaillancourt et al. 2002). In most of metabolisms of PAHs and heteroaromatic compounds (e.g., polychlorinated dibenzo- $p$-dioxins, dibenzofurans, carbazoles), catecholic metabolites are produced both in initial reaction and intermediary metabolisms by different type of dioxygenase. Inactivation of corresponding enzyme reduces efficiency of toxic PAH removal and may increase toxic stress. Additional toxic effects of catecholic metabolites include redox cycling through quinine-quinol interconversion (Kim et al. 2004). Some bacteria (e.g., Mycobacterium vanbaalenii PYR-1) cope with this problem through the efficient removal of toxic quinones by reduction and Omethylation (Kim et al. 2004). In consideration of their strong chemical reactivities, various $o$-hydroxy aromatic aldehydes are additional candidates of toxic metabolites. In strain $\mathrm{C} 4$, most of the above metabolites were not detected. Rapid transformation of these metabolites suggests that strain C4 may have specified enzymes for corresponding metabolites.

Oxidative stresses are common in various bacteria under harmful conditions (e.g., starvation, PAHs, toxins, oxidants), characterized by upregulation of responsive proteomes (Lee et al. 2007). In addition to such enzymes, the regulation of oxidant scavenging primary metabolite biosynthesis or decreasing the level of possible precursors of oxidant production may be another way to respond to oxidative stress (e.g., ascorbic acid). In strain $\mathrm{C} 4$, cysteine level was decreased during phenanthrene metabolism. Although cystein is an important precursor of various intracellular reducing equivalents (e.g., glutathione, thoredoxin), free cysteine can increase oxidative deoxyribonucleic acid damage through the enhancement of the Fenton reaction (Gusarov and Nudler 2005; Park and Imlay 2003). Decreas- 
ing free cysteine level in strain $\mathrm{C} 4$ may be a response to alleviate oxidative stress during phenanthrene metabolism. In addition to such metabolic adaptation to oxidative stresses, it is well known that PAH-degrading bacteria can efficiently remove various oxidants through the upregulation of superoxide dismutase or other equivalent enzymes (Lee et al. 2007).

Changes of cellular fatty acid and/or mycolic acid profile have been reported from several PAH-degrading bacteria (Mrozik et al. 2005; Tsitko et al. 1999; Wick et al. 2002, 2003). These adaptations usually provide bacterial cells to change their membrane fluidity with concomitant regulation of harmful chemicals flux. In usual, a shift to more hydrophobic or saturated rather than unsaturated fatty acids is a common response during the metabolism of less bioavailable/toxic xenobitics (Wick et al. 2002, 2003). Several possible advantages through these adaptations have been suggested, including enhancement of bioavailability of PAHs and/or confinement of toxic PAHs in/over the membrane. In strain $\mathrm{C} 4$, accumulation of cyclopropane fatty acid (C18) was the most characteristic change under the phenanthrene degradation condition. It is not clear whether the accumulation of this specific fatty acid modulates the membrane fluidity or hydrophobicity. However, the shift of other fatty acids to more hydrophobic analogues was coincided well with other bacterial species (Mrozik et al. 2005; Tsitko et al. 1999; Wick et al. 2002, 2003).

PHAs are biodegradable polymers, found in various bacterial species. These compounds are usually comprised with short-chain 3-hydroxyalkanoic acids (e.g., 3HB, 3HP, etc.). Although the exact biological role is not clear, PHAs are generally considered as reservoirs of excess carbon (Anderson and Dawes 1990). It is well known that various rhizobial bacteria, including Sinorhizobium spp., produce PHAs (e.g., Lakshman and Shamala 2006). From the analysis, it was clear that strain $\mathrm{C} 4$ can also produce PHAs from several different carbon sources. The monomeric composition was almost similar with other Sinorhizobium spp. (Lakshman and Shamala 2006). No significant changes were observed in the monomeric compositions from different carbon sources (Table 2). Because flux of PHAs is highly dependent on nutritional status of microorganisms, limited carbon supplementation is usually accompanied by PHA degradation, rather than their biosyntheses (Ruiz et al. 2001). Inverse relationships between the levels of PHAs and polyphosphate and nucleotides were reported (Ruiz et al. 2001). In strain C4, PHA level during phenanthrene degradation was much lower than that of other readily bioavailable carbon sources. Low concentration of PHAs in strain C4, grown with phenanthrene, suggest that the carbon supply from phenanthrene in a readily bioavailable form (e.g., pyruvate, sugars etc) may be much more reduced than other natural carbons. Limited bioavailability of PAHs and slow transformation to the metabolites in primary metabolism may be the cause of inhibition of PHA biosynthesis during phenanthrene degradation. It is noteworthy that de novo biosynthesis of fatty acid and PHAs shares common intermediates, ketoacyl-acyl-carrierproteins (Nomura et al. 2004; Rehm et al. 2001). Because of the possible competition between the two metabolic pathways, changes in fatty acid profiles may be interconnected with PHA contents. Specific type of transacylase $(\mathrm{PhlG})$ is an enzyme of metabolic divergence between the biosyntheses of fatty acid and PHAs (Rehm et al. 2001). However, it is not clear which metabolic factors or genes determine the divergence between two metabolic pathways.

In summary, large metabolomic differences of strain $\mathrm{C} 4$ have been observed between the cultures with phenanthrenen and natural carbon sources. The change includes TCA cycle, pyruvate metabolism, cofactor biosynthesis, fatty acid compositions, and PHA biosynthesis. Detailed information of metabolic adaptation may provide better understanding of this environmentally relevant bacterium to enhance or modify for specific purposes. However, there remain several questions. For example, chemical compositions of natural matrices (e.g., soils or pore water in soil environment) may be different from the medium in this study, and the metabolomic responses may also be deviated from the current studies. Because of the complexity of microbial communities, concentrations of some toxic metabolites may also be different from those of closed systems, as in this study. Further studies, including more close mimics with the natural environment and genomics/ proteomics, will give more detailed understanding of metabolic adaptation of strain $\mathrm{C} 4$.

Open Access This article is distributed under the terms of the Creative Commons Attribution Noncommercial License which permits any noncommercial use, distribution, and reproduction in any medium, provided the original author(s) and source are credited.

\section{References}

Aeckersberg F, Rainey FA, Widdel F (1998) Growth, natural relationships, cellular fatty acids and metabolic adaptation of sulfate-reducing bacteria that utilize long-chain alkanes under anoxic conditions. Arch Microbiol 170:361-369

Aitken MD, Stringfellow WT, Nagel RD, Kazunga C, Chen SH (1998) Characteristics of phenanthrene-degrading bacteria isolated from soils contaminated with polycyclic aromatic hydrocarbons. Can J Microbiol 44:743-752

Anderson AJ, Dawes EA (1990) Occurrence, metabolism, metabolic role, and industrial uses of bacterial polyhydroxyalkanoates. Microbiol Rev 54:450-472 
Armstrong B, Hutchinson E, Unwin J, Fletcher T (2004) Lung cancer risk after exposure to polycyclic aromatic hydrocarbons: a review and meta-analysis. Environ Health Perspect 112:970 978

Barsch A, Patschkowski T, Niehaus K (2004) Comprehensive metabolite profiling of Sinorhizobium meliloti using gas chromatographymass spectrometry. Funct Integr Genomics 4:219-230

Bastiaens L, Springael D, Wattiau P, Harms H, deWachter L, Verachtert H, Diels L (2000) Isolation of adherent polycyclic aromatic hydrocarbon (PAH)-degrading bacteria using $\mathrm{PAH}-$ sorbing carriers. Appl Environ Microbiol 66:1834-1843

Berger E, Ramsay BA, Ramsay JA, Chavarie C, Braunegg G (1989) PHB recovery by hypochlorite digestion. Biotechnol Tech 3:227232

Case I, de Lorenzo V (2005) Genetically modified organisms for the environment: stories of success and failure and what we have learned from them. Int Microbiol 8:213-222

Chang H-K, Zylstra GJ (1999) Role of quinolinate phosphoribosyl transferase in degradation of phthalate by Burkholderia cepacia DBO1. J Bacteriol 181:3069-3075

Damaj M, Ahmad D (1996) Biodegradation of polychlorinated biphenyls by rhizobia: a novel finding. Biochem Biophys Res Commun 218:908-915

Denef VJ, Park J, Tsoi TV, Rouillard JM, Zhang H, Wibbenmeyer JA, Verstraete W, Gulari E, Hashsham SA, Tiedje JM (2004) Biphenyl and benzoate metabolism in a genomic context: Outlining genome-wide metabolic networks in Burkholderia xenovorans LB400. Appl Environ Microbiol 70:4961-4970

Denef VJ, Patrauchan MA, Florizone C, Park J, Tsoi TV, Verstraete W, Tiedje JM, Eltis LD (2005) Growth substrate and phase specific expression of biphenyl, benzoate and $\mathrm{C} 1$ metabolic pathways in Burkholderia xenovorans LB400. J Bacteriol 187:7996-8005

Eom IC, Rast C, Veber AM, Vasseur P (2007) Ecotoxicity of a polycyclic aromatic hydrocarbon $(\mathrm{PAH})$-contaminated soil. Ecotoxicol Environ Saf 67:190-205

Frassinetti S, Setti L, Corti A, Farrinelli P, Montevecchi P, Vallini G (1998) Biodegradation of dibenzothiophene by a nodulating isolate of Rhizobium meliloti. Can J Microbiol 44:289-297

Galibert F, Finan TM, Long SR, Puhler A, Abola P, Ampe F, BarloyHubler F, Barnett MJ, Becker A, Boistard P, Bothe G, Boutry M, Bowser L, Buhrmester J, Cadieu E, Capela D, Chain P, Cowie A, Davis RW, Dreano S, Federspiel NA, Fisher RF, Gloux S, Godrie T, Goffeau A, Golding B, Gouzy J, Gurjal M, Hernandez-Lucas I, Hong A, Huizar L, Hyman RW, Jones T, Kahn D, Kahn ML, Kalman S, Keating DH, Kiss E, Komp C, Lelaure V, Masuy D, Palm C, Peck MC, Pohl TM, Portetelle D, Purnelle B, Ramsperger U, Surzycki R, Thebault P, Vandenbol M, Vorholter FJ, Weidner S, Wells DH, Wong K, Yeh KC, Batut J (2001) The composite genome of the legume symbiont Sinorhizobium meliloti. Science 293:668-672

Gusarov I, Nudler E (2005) NO-mediated cytoprotection: instant adaptation to oxidative stress in bacteria. Proc Natl Acad Sci USA 102:13855-1380

Ishii N, Nakahigashi K, Baba T, Robert M, Soga T, Kanai A, Hirasawa T, Naba M, Hirai K, Hoque A, Ho PE, Kakazu Y, Sugawara K, Igarashi S, Harada S, Masuda T, Sugiyama N, Togashi T, Hasegawa M, Takai Y, Yugi K, Arakawa K, Iwata N, Toya Y, Nakayama Y, Nishioka T, Shimizu K, Mori H, Tomita M (2007) Multiple high-throughput analyses monitor the response of $E$. coli to perturbations. Science 316:593-597

Keum YS, Seo JS, Hu Y, Li QX (2006) Degradation pathways of phenanthrene by Sinorhizobium sp. C4. Appl Microbiol Biotechnol 71:935-941

Kim Y-H, Moody JD, Freeman JP, Brezna B, Engesser K-H, Cerniglia CE (2004) Evidence for the existence of PAH-quinone reductase and catechol-O-methyltransferase in Mycobacterium vanbaalenii PYR-1. J Ind Microbiol Biotechnol 31:507-516

Kweon O, Kim S-J, Jones RC, Freeman JP, Adjei MD, Edmondson RD, Cerniglia CE (2007) A polyomic approach to elucidate the fluoranthene-degradative pathway in Mycobacterium vanbaalenii PYR-1. J Bacteriol 189:4635-4647

Lakshman K, Shamala TR (2006) Extraction of polyhydroxyalkanoate from Sinorhizobium meliloti cells using Microbispora sp culture and its enzymes. Enz Microbial Technol 39:1471-1475

Lee S-E, Seo JS, Keum YS, Lee KJ, Li QX (2007) Fluoranthene metabolism and associated proteins in Mycobacterium sp. JS14. Proteomics 7:2059-2069

Mashego MR, Rumbold K, Mey MD, Vandamme E, Soetaert W, Heijnen JJ (2007) Microbial metabolomics: past, present and future methodologies. Biotechnol Lett 29:1-16

Mrozik A, Labuzek S, Piotrowska-Seget Z (2005) Changes in fatty acid composition in Pseudomonas putida and Pseudomonas stutzeri during naphthalene degradation. Microbiol Res 160: $149-157$

Nomura CT, Taguchi K, Taguchi S, Doi Y (2004) Coexpression of genetically engineered 3-ketoacyl-ACP synthase III (fabH) and polyhydroxyalkanoate synthase (phaC) genes leads to shortchain-length-medium-chain-length polyhydroxyalkanoate copolymer production from glucose in Escherichia coli JM109. Appl Environ Microbiol 70:999-1007

Park S, Imlay JA (2003) High levels of intracellular cysteine promote oxidative DNA damage by driving the Fenton reaction. J Bacteriol 185:1942-1950

Parnell JJ, Park JH, Denef V, Tsoi T, Hashsham S, Quensen J III, Tiedje JM (2006) Coping with polychlorinated biphenyl (PCB) toxicity: physiological and genome-wide responses of Burkholderia xenovorans LB400 to PCB-mediated stress. Appl Environ Microbiol 72:6607-6614

Rehm BHA, Mitsky TA, Steinbüchel A (2001) Role of fatty acid de novo biosynthesis in polyhydroxyalkanoic acid (PHA) and rhamnolipid synthesis by Pseudomonads: establishment of the transacylase (PhaG)-mediated pathway for PHA biosynthesis in Escherichia coli. Appl Environ Microbiol 67:3102-3109

Robertson DG (2005) Metabonomics in toxicology: a review. Toxicol Sci 85:809-822

Ruiz JA, López NI, Fernández RO, Méndez BS (2001) Polyhydroxyalkanoate degradation is associated with nucleotide accumulation and enhances stress resistance and survival of Pseudomonas oleovorans in natural water microcosms. Appl Environ Microbiol $67: 225-230$

Sasser M (2006) Bacterial identification by gas chromatographic analysis of fatty acids methyl esters (GC-FAME). Technical note no. 101. Microbial ID, Newark, DE

Scott DE, Ciulli A, Abell C (2007) Coenzyme biosynthesis: enzyme mechanism, structure and inhibition. Nat Prod Rev 24:1009-1026

Tsitko IV, Zaitsev GM, Lobanok AG, Salkinoja-Salonen MS (1999) Effect of aromatic compounds on cellular fatty acid composition of Rhodococcus opacus. Appl Environ Microbiol 65:853-855

Vaillancourt FH, Labbe' G, Drouin NM, Fortin PD, Eltis LD (2002) The mechanism-based inactivation of 2,3-dihydroxybiphenyl 1,2dioxygenase by catecholic substrates. J Biol Chem 277: 2019-2027

Weckwerth W (2003) Metabolomics in system biology. Ann Rev Plant Biol 54:669-689

Wick LY, Wattiau P, Harms H (2002) Influence of the growth substrate on the mycolic acid profiles of Mycobacteria. Environ Microbiol 4:612-616

Wick LY, Pelz O, Bernasconi SM, Andersen N, Harms H (2003) Influence of the growth substrate on ester-linked phospho- and glycolipid fatty acids of PAH-degrading Mycobacterium sp. LB501T. Environ Microbiol 5:672-680 